\title{
CLOUDY modeling of weird Far-IR emission in the central zone of the Helix Nebula
}

\author{
Adrienne Dove ${ }^{1}$ and Angela Speck ${ }^{1}$ \\ ${ }^{1}$ Department of Physics \& Astronomy, University of Missouri, Columbia, MO 65201, USA \\ email: ard3k5@mizzou.edu
}

\begin{abstract}
We present the results of CLOUDY modeling of the central zone of the Helix Nebula. Our aim was to match the observed, excess far-infrared emission. Our best fit models fill the central zone with $1 \mu \mathrm{m}$-sized grains, with a gas-to-dust ratio of 500 , where precise composition is unimportant. We speculate on the origin of these grains.
\end{abstract}

Keywords. (ISM:) planetary nebulae: individual (NGC 7293)

\section{Introduction}

The central zone of the Helix Nebula appears as a hole in the classic optical image, yet has been found to produce both He II emission (O'Dell 1998) and [OIV] emission (Leene $\&$ Pottasch 1987). Strong emission has been observed at $60-100 \mu \mathrm{m}$, but is not detected in the mid-IR (Cox 1998), or at 160 or $180 \mu \mathrm{m}$; this emission is suspected to be due to cool dust grains.

\section{Methods and Results}

We modeled the emission from a spherically symmetric photo-ionized nebula using CLOUDY version 05.07 (Ferland 1998), varying only the gas-to-dust ratio and the nature of the grains (Table 1). Previous models of dust in the Helix have assumed an ISM dust composition. However, given that the progenitor of the Helix is believed to be a $6.5 \mathrm{M}_{\odot}$ O-rich AGB star (Henry et al. 1999), the dust is unlikely ISM-like. We therefore tested the effect of changing the nature of the dust. Figure $1 b$ shows that the precise composition of the dust is not a critical factor in determining the continuum emission.

Our best-fit model (Figure 1) indicates that the central zone is filled with approximately $1 \mu \mathrm{m}$-sized grains, with a gas-to-dust ratio of 500 . To place a limit on the spread of this distribution, we constructed models around this central size. A power law $\left(\mathrm{a}^{-3.5}\right)$ distribution with a range of $0.5-2 \mu \mathrm{m}$ fits closely, as does a Gaussian distribution of grain sizes centered at $1 \mu \mathrm{m}$, with a FWHM of up to $0.6 \mu \mathrm{m}$.

\section{Conclusions}

Our models have produced a spectrum for approximately $1 \mu \mathrm{m}$-sized grains that fits well with the observed SED, with the result being fairly composition independent. These

Table 1. Model parameters

\begin{tabular}{lc}
\hline Parameters & Variations \\
\hline Gas-to-dust ratio & $50,100,333,500,1000$ \\
Single size distribution & $\mathrm{a}_{\min }=0.05 \mu \mathrm{m}$ (for all) \\
MRN size distribution & $\mathrm{a}_{\max }=0.1,1.0,5.0 \mu \mathrm{m}$ \\
Composition & astronomical silicate, graphite, or both \\
\hline
\end{tabular}




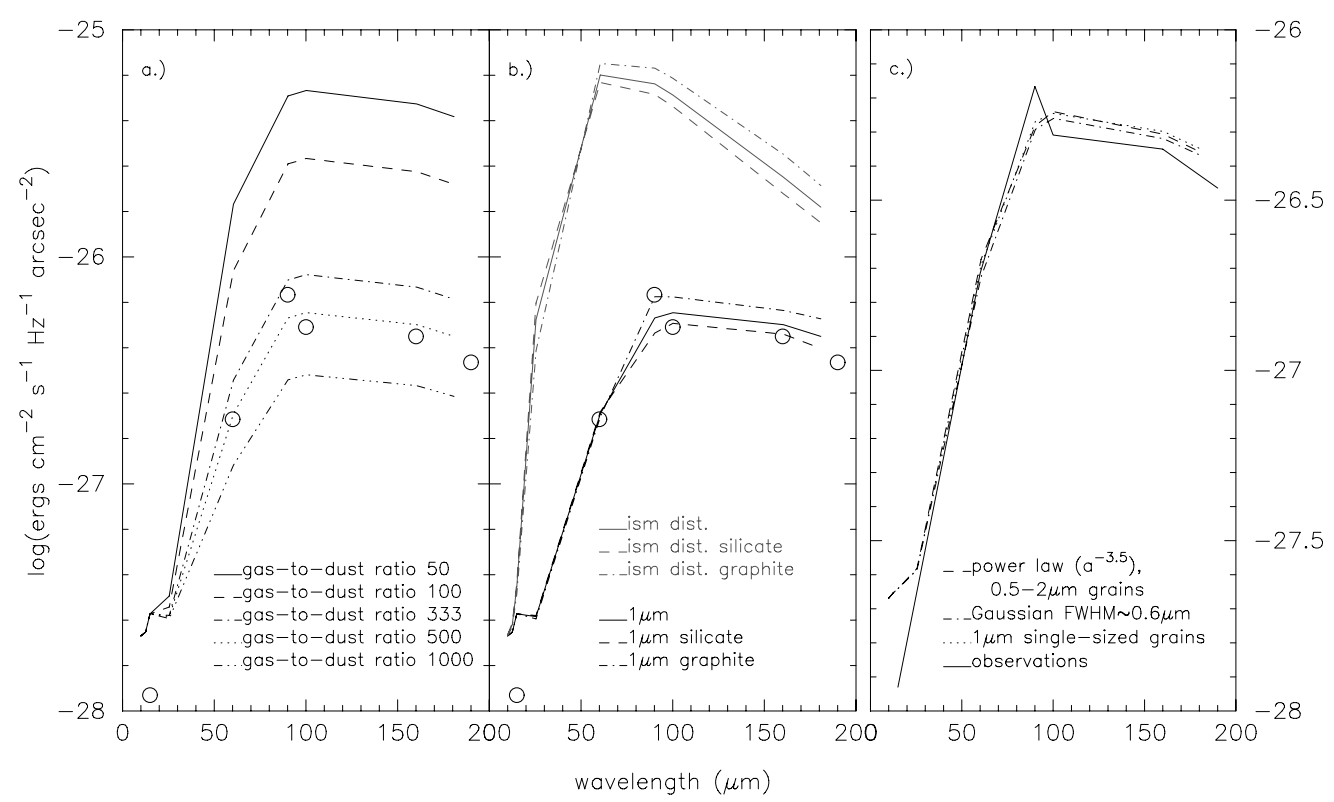

Figure 1. a) The effect of gas-to-dust ratio on the SED; (b) The effect of dust composition (open circles represent observational SED) (c) The observed SED of the central zone with the best fit models.

models demonstrate that we can account for this emission simply by changing the grain size distribution, while having little effect on other parameters.

We attempted to determine why such a narrow-range size distribution should exist, and found that the combined effects of radiation pressure and P-R drag should remove both small and large grains from the central region. This indicates that the dust grains in the central zone need to be constantly replenished. One possible dust source may be the slow destruction of cometary knots by the ionizing radiation. While grains smaller than $1 \mu \mathrm{m}$ will certainly be pushed outwards, P-R drag should lead to a distribution of grains which are much larger than can fit the observed SED. This implies that a.) the dust coming off of the knots has a high albedo; b.) very few grains larger than $1 \mu \mathrm{m}$ can be produced in the destruction of the knots (as they would remain in the central region longer than the smaller grains); or c.) the $1 \mu \mathrm{m}$-sized grains come together to form larger, fluffy grains with lower density and higher albedo that behave spectrally like their smaller components.

\section{References}

Cox, P. 1998, ApJ 495, L23

Ferland, G. J. et al. 1998 PASP 110, 761

Henry, R. B. C., Kwitter, K. B., \& Dufour, R. J. 1999 ApJ 517, 782

Leene, A., \& Pottasch, S. R. 1987 A\&̈A 173, 145

O'Dell, C. R. 1998 AJ 116, 1346

Speck, A. K., et al. 2002 AJ 123, 346 